\title{
Sorafenib suppresses the epithelial- mesenchymal transition of hepatocellular carcinoma cells after insufficient radiofrequency ablation
}

Shuying Dong ${ }^{1 \dagger}$, Jian Kong ${ }^{1 \dagger}$, Fandong Kong ${ }^{3,4}$, Jinge Kong ${ }^{3,4}$, Jun Gao ${ }^{1}$, Liang Ji², Bing Pan ${ }^{2}$, Lian Chen ${ }^{5}$, Lemin Zheng ${ }^{2^{*}}$ and Wenbing Sun ${ }^{* *}$

\begin{abstract}
Background: Epithelial-mesenchymal transition (EMT) played an important role in the progression of hepatocellular carcinoma (HCC) after insufficient radiofrequency ablation (RFA). However, whether sorafenib could be used to suppress the EMT of HCC after insufficient RFA and further prevent the progression of residual HCC remains poorly unknown.

Methods: Insufficient RFA was simulated using a water bath $\left(47^{\circ} \mathrm{C}\right.$, 10, 15, 20 and 25 min gradually). MTT assay and transwell assay were used to evaluate the effects of sorafenib on viability, migration and invasion of HepG2 and SMMC7721 cells after insufficient RFA in vitro. After insufficient RFA, the molecular changes in HCC cells with the treatment of sorafeinb were evaluated using western blot and ELISAs. An ectopic nude mice model was used to evaluate the effect of sorafenib on the growth of HepG2 cells in vivo after insufficient RFA.

Results: HepG2 and SMMC7721 cells after insufficient RFA (named as HepG2-H and SMMC7721-H) exhibited enhanced viability, migration and invasion in vitro. Sorafenib inhibited the enhanced viability, migration and invasion of HepG2 and SMMC7721 cells after insufficient RFA. Molecular changes of EMT were observed in HepG2-H and SMMC7721-H cells. Sorafenib inhibited the EMT of HepG2-H and SMMC7721-H cells. HepG2-H cells also exhibited larger tumor size in vivo. Higher expression of PCNA, Ki67, N-cadherin, MMP-2 and MMP-9, was also observed in HepG2-H tumors. Sorafenib blocked the enhanced growth of HepG2 cells in vivo after insufficient RFA.
\end{abstract}

Conclusions: Sorafenib inhibited the EMT of HCC cells after insufficient RFA, and may be used to prevent the progression of HCC after RFA.

Keywords: Hepatocellular carcinoma, Insufficient radiofrequency ablation, Epithelial-mesenchymal transition, Sorafenib

\section{Background}

Hepatocellular carcinoma (HCC) is the fifth most common tumor worldwide and is the third most common cause of cancer-related death [1]. Radiofrequency ablation (RFA) is emerging as an effective local treatment for curative intent in patients with cirrhosis and HCC smaller

\footnotetext{
* Correspondence: Zheng|@bjmu.edu.cn; cyhswb@qq.com

${ }^{\dagger}$ Equal contributors

${ }^{2}$ School of Basic Medical Sciences, and Key Laboratory of Molecular Cardiovascular Sciences of Ministry of Education, The Institute of Cardiovascular Sciences and Institute of Systems Biomedicine, Peking University Health Science Center, Beijing 100191, China

${ }^{1}$ Department of Hepatobiliary Surgery, Beijing Chao-yang Hospital, Capital Medical University, Beijing 100043, China

Full list of author information is available at the end of the article
}

than $3 \mathrm{~cm}$ in diameter [2]. However, the major problem with RFA is its difficulty in achieving complete tumor destruction [3], and several cases of rapid and aggressive recurrence of HCC after RFA have been reported [4-6].

Several mechanisms have been proposed to explain the phenomenon of progression of HCC after insufficient RFA [7-12]. Epithelial-mesenchymal transition (EMT) is a complex process, involving dissolution of cell-cell junctions and loss of apical-basolateral polarity, resulting in transition of epithelial cells into migratory mesenchymal cells with invasive properties [13]. Yoshida et al. reported that sublethal heat treatment skews HCC cells toward EMT and transforms them to a progenitor- 
like, highly proliferative cellular phenotype in vitro and in vivo, which driven significantly by p46Shc-ERK1/2, and suboptimal RFA accelerates HCC growth and spread by transiently inducing an EMT-like, more aggressive cellular phenotype [12]. Our previous study established a model simulating insufficient RFA in vitro and showed that insufficient RFA could directly promote the invasiveness and metastasis of HCC cells and the EMT of HCC cells through Akt and ERK signaling pathways [11]. However, there are no researches about exploring effectual approaches to prevent the progression of HCC after insufficient RFA.

Sorafenib is the first and only molecular targeted therapy approved for use in HCC by the U.S. Food and Drug Administration in 2007. Currently, sorafenib is used as a standard treatment for patients with advanced HCC. Sorafenib inhibited the EMT induced by transforming growth factor $\beta 1$ in mouse hepatocytes and hepatocyte growth factor in HCC $[14,15]$. Sorafenib ameliorated bleomycin-induced pulmonary fibrosis through inhibiting EMT and fibroblast [16], and inhibited EMT in human lung epithelial cells [17]. Sorafenib was also used to suppress postsurgical recurrence and metastasis of $\mathrm{HCC}$ in an orthotopic mouse model, indicating that sorafenib had a potential application in early-stage HCC patients who have undergone hepatectomy with curative intention [18]. Apart from its successful application in patients with advanced, unresectable $\mathrm{HCC}$, however, the use of sorafenib in patients with early-stage HCC is largely untested; this is especially true for patients who are considered as appropriate candidates for curative intervention. Whether sorafenib could be used to suppress the EMT of HCC after insufficient RFA and further prevent the progression of residual HCC remains poorly unknown.

In the present study, we established a simulated model to understand the recurrence of aggressive HCC after insufficient RFA. We investigated the effects of sorafenib on cell growth, migration and invasion of HCC cell lines (HepG2 and SMMC7721) after insufficient RFA in vitro. Furthermore, we analyzed the influences of sorafenib on changes of epithelial and mesenchymal markers, and Akt and ERK1/2 signaling pathways involved in the process in HCC cells after insufficient RFA. We also performed in vivo experiments to study the effect of sorafenib on the growth of HCC cells after insufficient RFA in a BALB/c nu/nu mice model.

\section{Methods}

\section{Ethics statement}

All animal experiments were approved by Animal Care Committee of Capital Medical University and performed in accordance with the institutional guideline. All sections of this report adhere to the ARRIVE Guidelines for reporting animal research [19]. A completed ARRIVE guidelines checklist is included in Additional file 1.

\section{Reagents}

Sorafenib was kindly provided by Bayer Pharmaceuticals. 3(4, 5-dimethylthiazol-2-yl)-2, 5-diphenyltetrazolium bromide (MTT) was obtained from Sigma (Shanghai, China). Phospho-anti-Akt and phospho-anti-ERK1/2 antibodies were purchased from Cell signaling (Beverly, CA, USA). Anti-E-cadherin, $\mathrm{N}$-cadherin, vimentin, and snail were bought from Abcam (Cambridge, TX, USA). Anti- $\beta$-actin, PCNA, Ki67, MMP-2 and MMP-9 antibodies were obtained from Santa Cruz (Dallas, TX, USA). Enzyme-linked immunosorbent assay (ELISA) kits for E-cadherin, Ncadherin, MMP-2 and MMP-9 were purchased from RD (Minneapolis, MN, USA) and kits for vimentin and snail were from Bioss (Beijing, China).

\section{Cell culture}

HCC cell lines, HepG2 and SMMC7721, were from the American Type Culture Collection (ATCC) (Manassas, VA, USA). HCC cells were cultured in high-glucose Dulbecco's modified Eagle medium (DMEM) supplement with $10 \%$ heat-inactivated fetal bovine serum (FBS), 100 $\mathrm{U} / \mathrm{ml}$ penicillin and $100 \mu \mathrm{g} / \mathrm{ml}$ streptomycin (Life Technologies, Cergy Pontoise, France) at $37{ }^{\circ} \mathrm{C}$ in a humidified incubator with $5 \% \mathrm{CO}_{2}$.

\section{Heat treatment}

Insufficient RFA was simulated in vitro as described before [11]. Briefly, HCC cells were seeded into the 6-well plates $\left(5 \times 10^{4}\right.$ cells/well). After $24 \mathrm{~h}$, the plates were sealed and submerged in a water bath set to 37 or $47^{\circ} \mathrm{C}$ for $5 \mathrm{~min}$. Thereafter, cells were allowed to recover, and when the surviving populations reached $80 \%$ confluence, cells were propagated into the 6 -well plates and exposed to above heat treatment for $10 \mathrm{~min}$. Then the process was repeated and cells were sequentially exposed to above heat treatment for 15,20 and $25 \mathrm{~min}$. Cells surviving from the treatment of $47{ }^{\circ} \mathrm{C}$ for $25 \mathrm{~min}$ were designated as HepG2-H and SMMC7721-H cells.

\section{MTT assay}

Cell viability was analyzed using the MTT assay. Briefly, HCC cells were cultured in 96-well plates at a concentration of $3 \times 10^{3} /$ well, incubated for $24 \mathrm{~h}$, and treated with sorafenib. After 24,48 , or 72 h treatment, MTT solution was added to each well at a final concentration of $0.5 \mathrm{mg} / \mathrm{ml}$ and the cells were incubated for $4 \mathrm{~h}$. At the end of incubation, formazan crystals resulting from MTT reduction were dissolved by addition of $150 \mu \mathrm{l}$ dimethyl sulfoxide per well. The absorbance was measured at $570 \mathrm{~nm}$ using an automated ELISA plate reader. 


\section{Colony formation assay}

HCC cells $\left(1 \times 10^{3} /\right.$ well $)$ were seeded into 6-well dishes and allowed to grow for $24 \mathrm{~h}$. The cells were then incubated in the presence or absence of sorafenib for 2 weeks in complete medium. The colonies obtained were washed with PBS and fixed in $4 \%$ paraformaldehyde for $20 \mathrm{~min}$ at room temperature and then stained with crystal violet. The colonies were photographed under an inverted fluorescence microscope (Olympus IX51) equipped with an Olympus Qcolor 3 digital camera (Olympus). The colonies were counted from 6 random fields at $12.5 \times$ magnification.

\section{Migration and invasion assays}

Quantitative cell migration assays were performed using a modified Boyden chamber (Costar-Corning, New York, USA) with $8.0-\mu \mathrm{m}$ pore polycarbonate filter inserts in 24-well plates as described previously. Briefly, the lower chamber was filled with DMEM with $10 \% \mathrm{FBS}$, and HCC cells $\left(5 \times 10^{4}\right.$ cells/well $)$ in serum-free medium were added into the upper chamber. After $30 \mathrm{~min}$, sorafenib was added to the upper chamber. The cells were allowed to migrate for $24 \mathrm{~h}$ at $37{ }^{\circ} \mathrm{C}$. The non-migrated cells were removed from the upper surface of the membrane by scraping with a cotton swab, and the migrating cells were fixed with methanol, stained with crystal violet (Beyotime, Nantong, China) and photographed under an inverted fluorescence microscope (Olympus IX51) equipped with an Olympus Qcolor 3 digital camera (Olympus). Migration was assessed by counting the number of stained cells from 10 random fields at $200 \times$ magnification. Cell invasion assay was performed similarly, except that transwell inserts were matrigel-coated.

\section{Western blot analysis}

HCC cells were lysed with lysis buffer $[150 \mathrm{mM} \mathrm{NaCl}$, $50 \mathrm{mM}$ Tris- $\mathrm{HCl}$ (pH 8.0), 0.1 \% SDS, 1 \% Triton X100] containing protease and phosphatase inhibitor. Cell lysate protein content was determined using a Bicinchoninic acid (BCA) protein assay kit. Equivalent amounts of whole cell extracts were subjected to SDS-PAGE gel and transferred to nitrocellulose membranes. The membranes were blocked with $5 \%$ non-fat milk for $2 \mathrm{~h}$ and then incubated with respective primary antibody overnight at $4{ }^{\circ} \mathrm{C}$ followed by the incubation with the appropriate HRP-conjugated secondary antibody for $1.5 \mathrm{~h}$ at room temperature. Blots were visualized with an ECL detection kit (Pierce, USA) and analyzed using Quantity One 1-D Analysis Software (Bio-Rad, Hercules, USA).

\section{Enzyme-linked immunosorbent assay}

Cytokines secreted into the conditioned medium were quantified using ELISA kits according to the manufacturer's instructions. The concentration of cytokines was normalized to the total cellular protein using a BCA protein assay kit.

\section{Xenografts assay}

BALB/C nude mice (male, 4-5 weeks old) were obtained from Vital River (Beijing, China). Mice were maintained under pathogen-free conditions and housed in barrier facilities on a 12-h light/dark cycle, with food and water ad libitum. HepG2 and HepG2-H cells $\left(5 \times 10^{6}\right)$ were suspended in $200 \mu \mathrm{l}$ serum-free DMEM and matrigel (1:1) and then injected subcutaneously into the upper right flank region of 20 nude mice. After $1 \mathrm{w}$, mice were treated with sorafenib by oral route $(30 \mathrm{mg} / \mathrm{kg} /$ day $)$, or polyoxyethylenated castor oil as control every day for up to the $22^{\text {th }}$ day ( $\mathrm{n}=5$ each group). Tumor size was measured with a caliper rule every other day. The tumor volume was calculated as follows: TV $\left(\mathrm{mm}^{3}\right)=\left(\mathrm{L} \times \mathrm{W}^{2}\right)$ / 2 , where $\mathrm{L}$ was the longest and $\mathrm{W}$ the shortest radius of the tumor in millimeters. At the end of the experiments, mice were euthanized by cervical dislocation, blood samples were collected via cardiac puncture after the mice were anesthetized i.p. with $400 \mathrm{mg} / \mathrm{kg}$ chloral hydrate (SCRC, Shanghai, China), and tumor tissues were removed for fixation in the $4 \%$ paraformaldehyde for histologic examination and immunohistochemical staining.

\section{Immunohistochemical analysis}

Tissues were fixed in $4 \%$ paraformaldehyde and subsequently embedded in paraffin. Paraffin-embedded tissue sections were cut into standard $6 \mu \mathrm{m}$ sections, deparaffinaged in xylene and rehydrated through graded alcohol solutions. Antigen retrieval was performed $10 \mathrm{~min}$ at $92{ }^{\circ} \mathrm{C}$ in EDTA (10 mM, pH 8.0) in a water bath. Endogenous peroxidases were inactivated by immersing the sections in $0.3 \%$ hydrogen peroxide for $12 \mathrm{~min}$. The sections were blocked with $5 \%$ goat serum for $60 \mathrm{~min}$ at $37{ }^{\circ} \mathrm{C}$. The slides were incubated with primary antibodies for overnight at $4{ }^{\circ} \mathrm{C}$. Next, the slides were treated with appropriate HRP-conjugated secondary antibody for $40 \mathrm{~min}$ at $37{ }^{\circ} \mathrm{C}$ and then developed with 3,3'-diaminobenzidine. Finally, the slides were counterstained with hematoxylin and mounted. The slides were examined with Nikon Eclipse Ti microscope under a $200 \times$ objective.

\section{Statistical analysis}

All values are expressed as the mean \pm SEM. The data were analyzed using the ANOVA test. A P value of $<0.05$ was considered statistically significant. GraphPad Prism (GraphPad Software Inc., San Diego, California, USA) was used for these analyses. 


\section{Results}

Sorafenib inhibited the enhanced viability of HCC cells after insufficient RFA

First of all, HepG2 or SMMC7721 cells were treated with heat treatment for $5,10,15,20$ and $25 \mathrm{~min}$ gradually as described above. Cells surviving from the treatment of $47{ }^{\circ} \mathrm{C}$ for 25 min were designated as HepG2-H and SMMC7721-H cells when we could see the morphological changes. To evaluate the effects of sorafenib on HCC cells, all cells were treated with sorafenib for 24, 48 or $72 \mathrm{~h}$ at different concentrations. HepG2-H cells exhibited higher viability rate compared with HepG2 cells at 48 and 72 h (Fig. 1a). Sorafenib inhibited the viability rate of HepG2 and HepG2-H cells in a time- and dose- dependent manner (Fig. 1a). After the treatment of sorafenib (5 and $10 \mu \mathrm{M})$ for 24 and $48 \mathrm{~h}$ and sorafenib (2, 5 and $10 \mu \mathrm{M})$ for $72 \mathrm{~h}$, the distinction of viability rate between HepG2 and HepG2-H cells faded (Fig. 1a). To determine the long term growth ability, sorafenib was allowed to treated HCC cells for 2 weeks. HepG2-H cells had a higher number of colonies in comparison with HepG2 cells (Fig. 1b and c). Sorafenib also suppressed the colony formation of HepG2 and HepG2$\mathrm{H}$ cells in a dose-dependent manner. Similar results were also found in SMMC7721 and SMMC7721-H cells (Additional file 2: Figure S1).
Sorafenib inhibited the enhanced migration and invasion abilities of HCC cells after insufficient RFA

HepG2-H cells displayed enhanced migration and invasion abilities compared with HepG2 cells (Fig. 2). Sorafenib inhibited the migration and invasion abilities of HepG2 and HepG2-H cells (Fig. 2). Sorafenib (5 and $10 \mu \mathrm{M})$ eliminated the difference of migration ability between HepG2 and HepG2-H cells, and sorafenib (2, 5 and $10 \mu \mathrm{M}$ ) eliminated the difference of invasion ability between HepG2 and HepG2-H cells (Fig. 2). Similar results were also in SMMC7721 and SMMC7721-H cells (Additional file 2: Figure S2).

\section{Sorafenib suppressed the EMT of HCC cells after insufficient RFA}

HepG2-H and SMMC7721-H displayed a spindle shape with less cell-cell adhesion and increased formation of pseudopodia, and sorafenib inhibited the morphological changes above (Fig. 3a). To evaluate whether EMT had occurred in HepG2-H and SMMC7721-H cells, EMT markers were examined. Western blot and ELISA assay showed that significant reduction in E-cadherin expression and up-regulation of N-cadherin, vimentin, snail, MMP-2 and MMP-9 were found in HepG2-H (Figs. 3b and 4) and SMMC7721-H cells (Fig. 3b and Additional file 2: Figure S3). Sorafenib increased the expression of E-cadherin, and decreased the expression of N-cadherin,

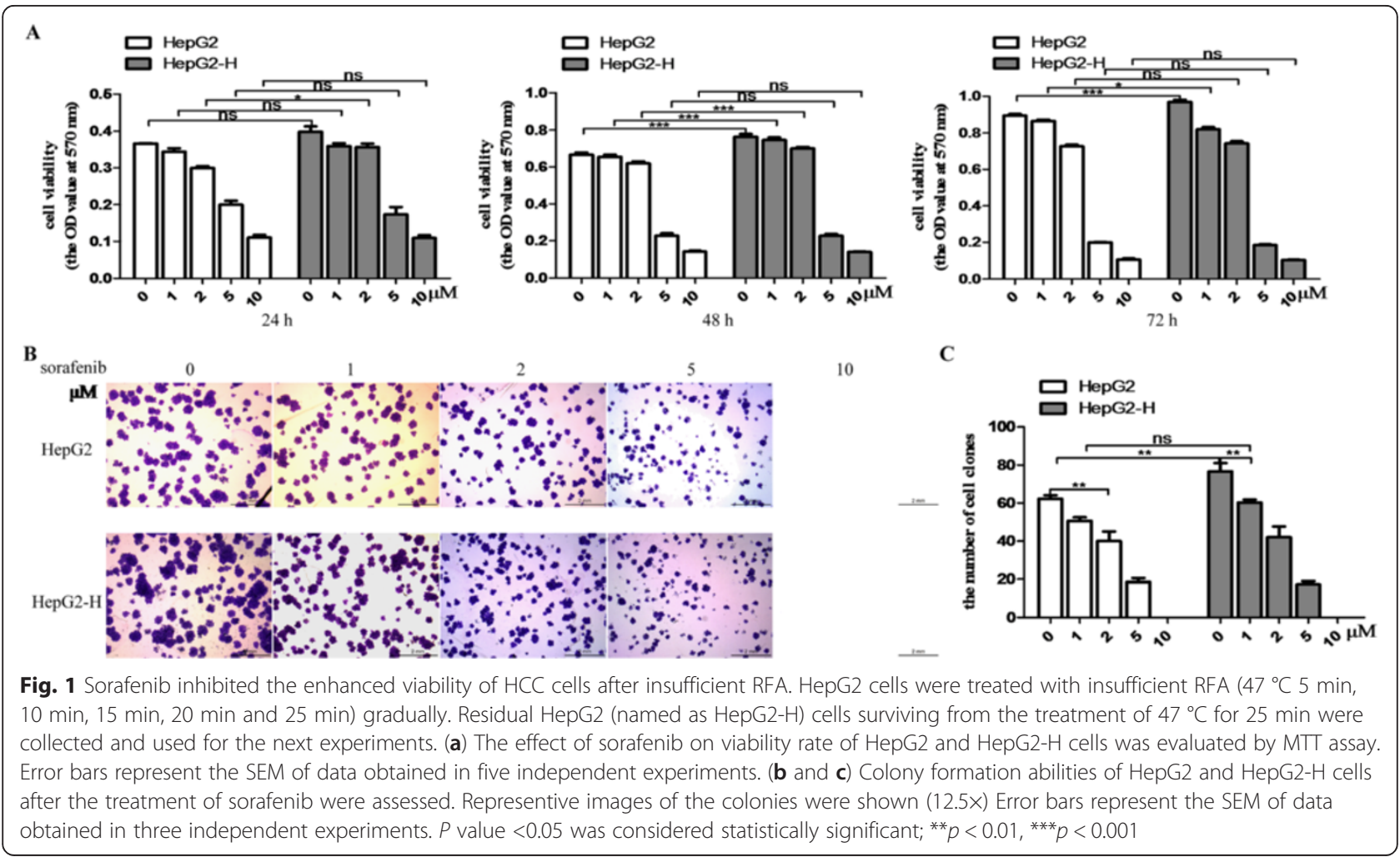




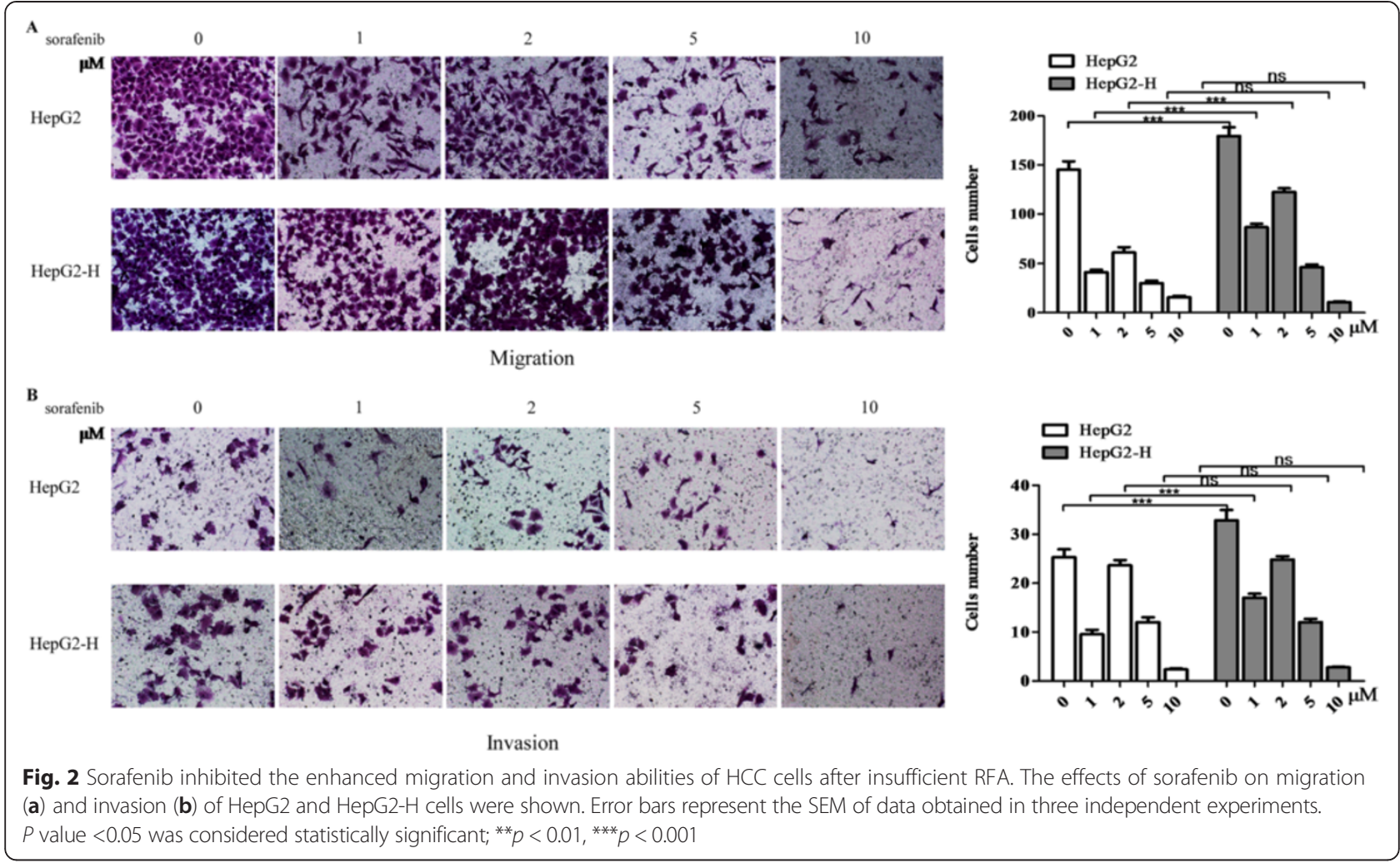

vimentin, snail, MMP-2 and MMP-9 in HCC cells in a dose-dependent manner (Figs. 3b, 4 and Additional file 2: Figure S3). To explore the signaling mechanisms involved in the sorafenib on EMT of HCC cells after insufficient RFA, we tested Akt and ERK1/2 signaling pathways. HepG2-H and SMMC7721-H cells showed significantly increased expression of p-Akt and p-ERK1/ 2 compared with HepG2 and SMMC7721 cells respectively (Figs. 3c, 4a and Additional file 2: Figure S3A). Furthermore, sorafenib inhibited the expression of $\mathrm{p}$-Akt and p-ERK1/2 in HCC cells in a dose-dependent manner (Figs. 3c, 4a and Additional file 2: Figure S3A).

Insufficient RFA enhanced the growth of HCC cells in vivo, and sorafenib blocked the process

HepG2-H cells showed increased tumor volume compared with HepG2 cells (Fig. 5a and b). Sorafenib suppressed the growth of HepG2 and HepG2-H cells (Fig. 5a and b). Sorafenib diminished the difference of tumor growth between HepG2 and HepG2-H cells (Fig. 5a and b). Significant increases of cell proliferation were observed in HepG2-H cells, and sorafenib inhibited the process (Fig. 5c). In addition, HepG2-H tumors showed decreased expression of E-cadherin and increased expression of N-cadherin, MMP-2 and MMP-9 compared with HepG2 tumors (Fig. 5c). And sorafenib increased the expression of E-cadherin, and decreased the expression of N-cadherin, MMP-2 and MMP-9 in HepG2 and HepG2-H tumors (Fig. 5c). However, there were no apparent changes in liver, heart, kidney, lung and body weight in the mice (Additional file 2: Figure S4).

\section{Discussion}

Local recurrences of $\mathrm{HCC}$ can progress rapidly after RFA, and EMT may involve in the progress. In the present study, we established a model simulating the HCC cells after insufficient RFA. And, we showed that sorafenib significantly suppressed the EMT of HCC after insufficient RFA in vitro and vivo. The finding may have a beneficial impact on the clinical practice of HCC therapy and especially on the radical intervention used for early-stage patients. Sorafenib may be applied to patients who have undergone RFA, and used to improve the prognosis via the effective inhibition of EMT.

Our previous study demonstrated that EMT occurred in SMMC7721 and Huh7 cells after insufficient RFA [11]. In this study, HepG2 cells were treated with insufficient RFA gradually as previous, and EMT also occurred. In another research, HepG2, Huh7 and HepG3B cells were exposed to $50{ }^{\circ} \mathrm{C}$ for $10 \mathrm{~min}$, and EMT occurred on day 5 after treatment, which was partly consistent with our results [12]. Our results further confirmed that insufficient RFA promoted the EMT of HCC cells. Furthermore, in our results, sorafenib suppressed the EMT of HCC cells after insufficient RFA, which was not ever reported before. 


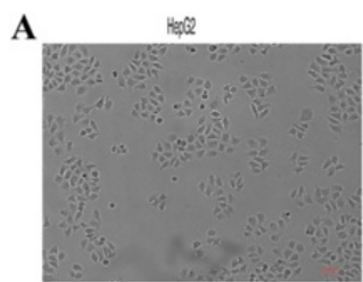

SMIC721

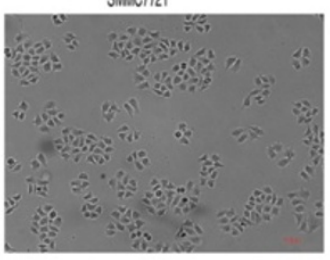

B

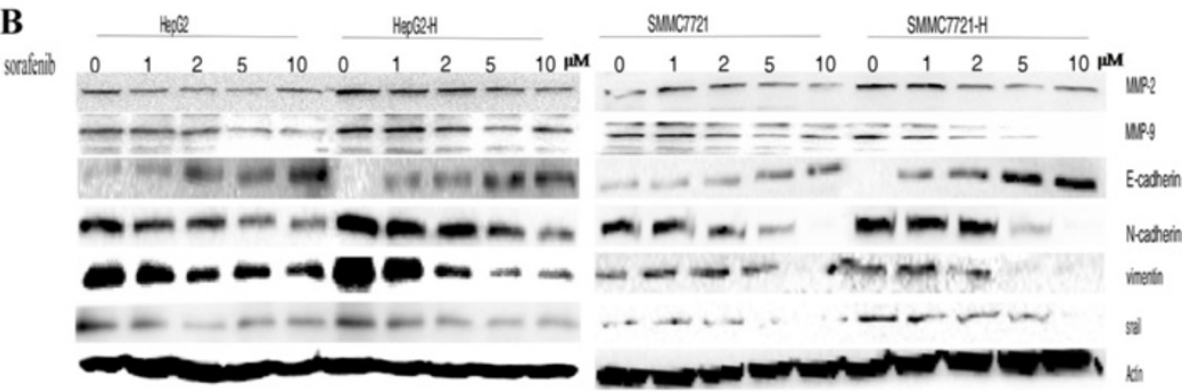

C

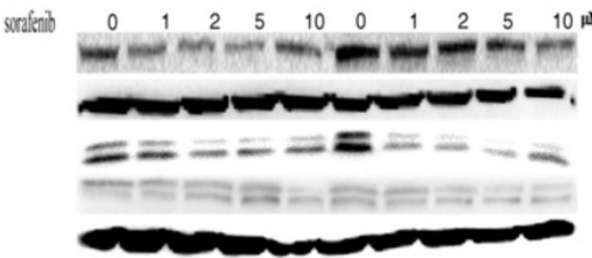

HepG2-H-sora

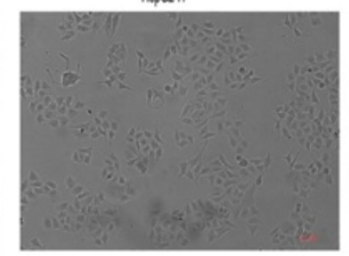

SMMC7721H
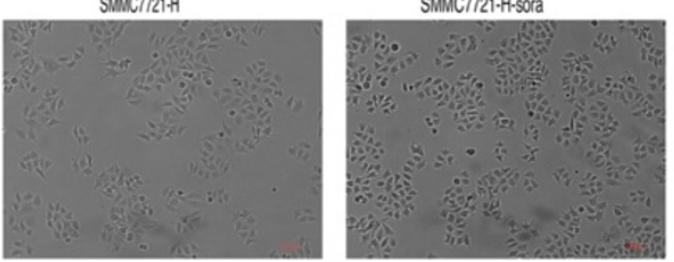

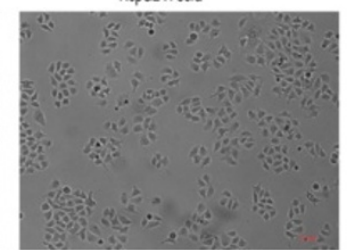

SMMC7721-H-sora

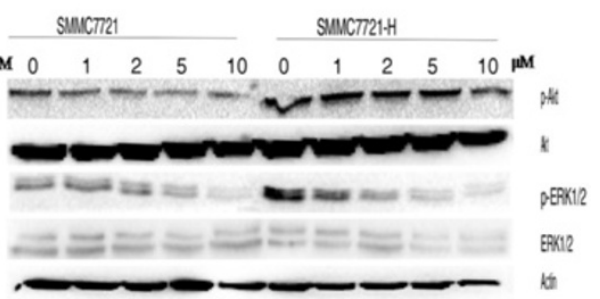

Fig. 3 Sorafenib suppressed the EMT of HCC cells after insufficient RFA. Sorafenib was used to treat HCC cells, the morphological changes of HepG2 and SMMC7721 after insufficient RFA were displayed (a) and western blot was used to determine the expression of MMP-2, MMP-9, E-cadherin, N-cadherin, vimentin, snail (b), p-Akt, Akt, p-ERK1/2 and ERK1/2 (c)

Recently, Akt and ERK signaling pathways have been reported to play an important role in EMT in HCC. MicroRNA-331-3p promoted proliferation and EMTmediated metastasis of $\mathrm{HCC}$ via the suppression of PHLPP-mediated de-phosphorylation of Akt [20]. Maelstrom promoted HCC metastasis by inducing EMT by way of Akt/GSK-3 $\beta$ pathways [21]. MicroRNA-21 suppressed PTEN and hSulf- 1 expression and promoted HCC progression through Akt/ERK pathways [22]. Akt and ERK pathways were also mediated the EMT of HCC cells after insufficient RFA [11], and sublethal heat treatment promoted EMT of HCC via ERK pathways [12]. Therefore, the inhibitors of Akt and ERK may be applied to suppress the EMT of HCC cells after insufficient RFA. However, all specific inhibitors of Akt are in preclinical trials $[23,24]$. Sorafenib was originally developed based on its inhibitory effect of Raf and receptor tyrosine kinase signaling, which further inhibited the RafMEK-ERK signaling pathway [25]. Sorafenib has been reported to suppress the growth and metastasis of HCC via Akt and ERK pathways [26-28], and inhibit EMT of HCC induced by growth factors [14, 15]. In the present study, sorafenib inhibited the up-regulation of p-Akt and p-ERK1/2 in HCC cells after insufficient RFA, and further down-regulated the increased expression of $\mathrm{N}$ cadherin, vimentin and snail, which resulted in enhanced abilities of migration and invasion in HCC cells after insufficient RFA.

Recently, sorafenib has been used to strengthen the curative effect of RFA in HCC. Fukuda $\mathrm{H}$ et al. reported that combination of sorafenib and RFA may be superior to standard RFA alone in the treatment of HCC tumors 


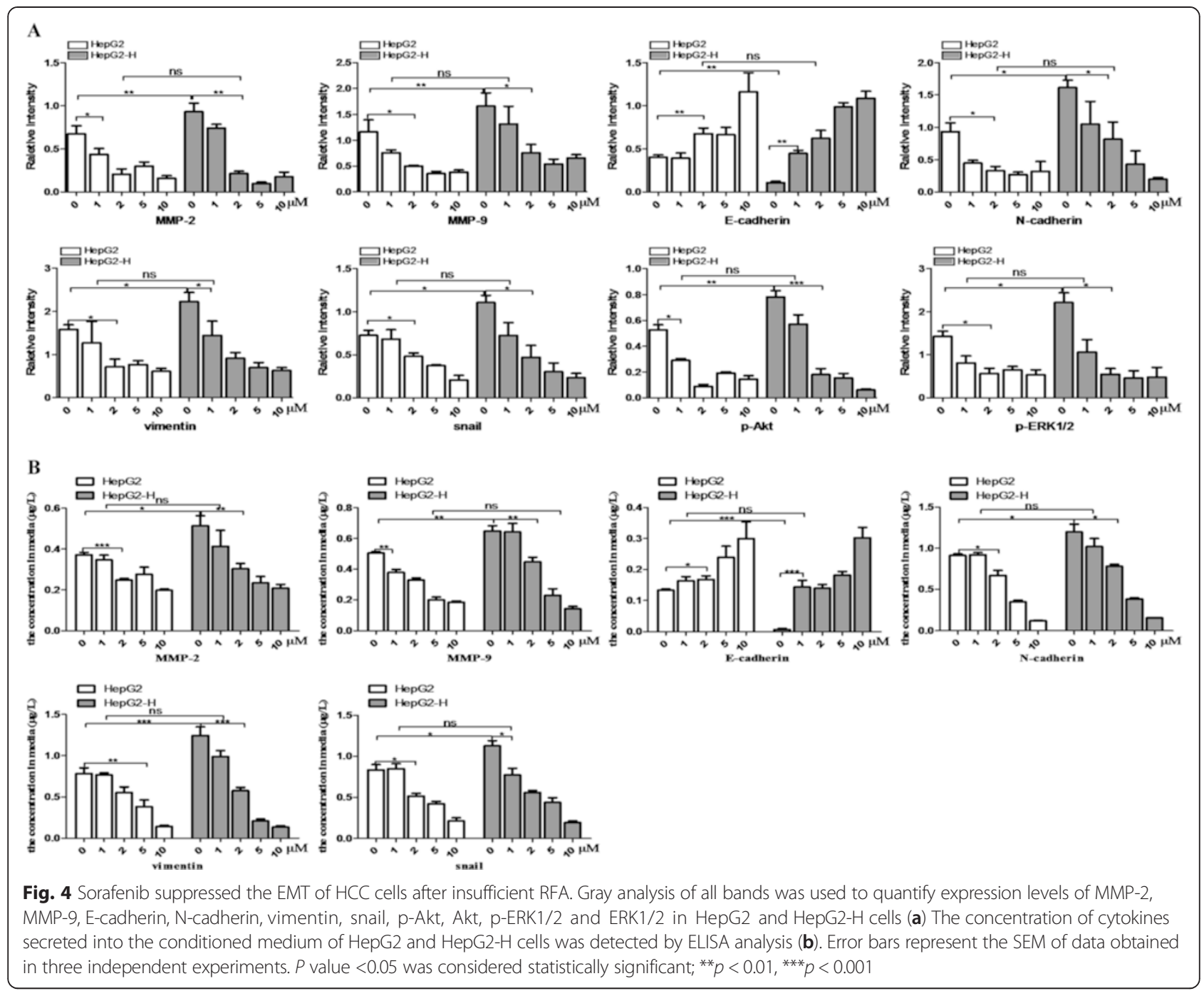

smaller than $3 \mathrm{~cm}$ in diameter [29]. Li Y et al. reported that the combination of sorafenib, transarterial chemoemboliztion and RFA proved both safe and effective in the treatment for unresectable HCC patients [30]. Another research demonstrated that sorafenib suppressed the rapid progress of $\mathrm{HCC}$ after insufficient ablation therapy in an experiment in vivo, which seemed like our research [31]. In their study, sorafenib was able to inhibit the up-regulated expression of HIF- $1 \alpha$ and VEGFA in a xenograft model after partial RFA. However, rapid progress of HCC after insufficient RFA was not observed, and further mechanisms involved in the progress were not investigated. According to the guidelines of the National Centre for the Replacement, Refinement and Reduction of Animals in Research (London, UK), in the animal experiment, we produced reasonable evidence to support our findings in vitro by stopping large studies which would result in a large augment in animal use. In our study, sorafenib inhibited the migration and invasion of HCC cells through the suppression of EMT after insufficient RFA in the simulated model. Furthermore, in the mice model, HCC cells after insufficient RFA exhibited larger tumor volume compared with HCC cells after control treatment, and sorafenib inhibited the growth of HCC cells and eliminated the difference of growth in HepG2-H and HepG2 cells. So sorafenib may be used to prevent the progression of HCC cells after insufficient RFA.

Sorafenib causes multiple human toxicities, including use-limiting anorexia, GI bleeds and hand-foot syndrome, which may lead to drug discontinuation or dose reduction that can decrease the potential life-prolonging benefits of sorafenib [32, 33]. In our research, sorafenib $(5 \mu \mathrm{M})$ significantly suppressed the enhanced abilities of migration and invasion of HCC cells associated with EMT after insufficient RFA in vitro, and sorafenib (30 mg/ $\mathrm{kg} /$ day) inhibited the growth of HCC cells after insufficient RFA in vivo without more toxicity for mice. However, in the present study, we just used the simulated model which 


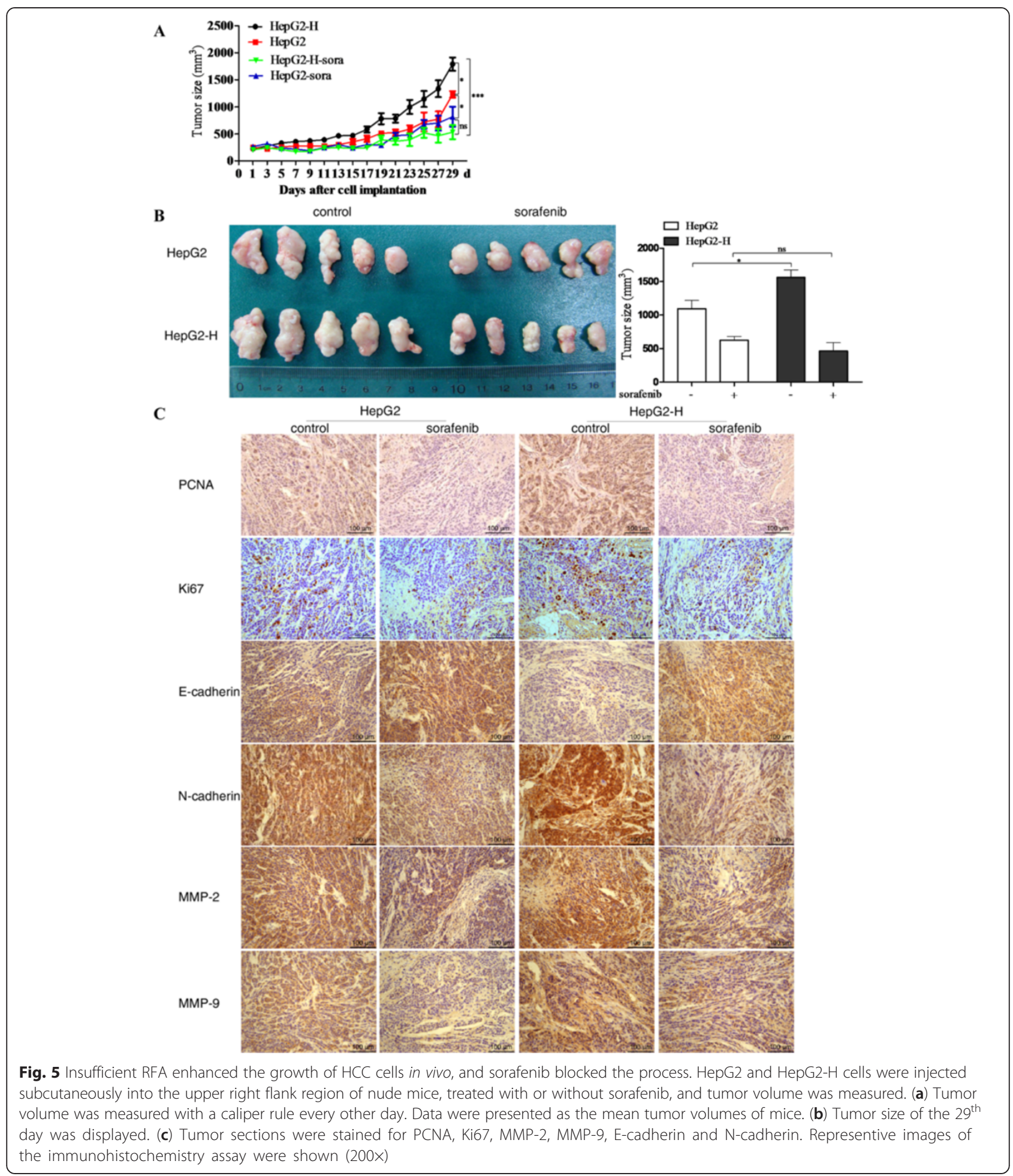

may not be representative of the progression of residual HCC tumor after insufficient RFA effectively and only provided the preliminary basis for the application of sorafenib to prevent the progression of HCC cells after RFA, and more mechanisms involved in the progression and clinical trials should be investigated in the future.

\section{Conclusions}

All in all, the study focused on a simulated model to understand the recurrence of aggressive HCC after insufficient RFA. This study demonstrated that insufficient RFA promoted the EMT of HCC cells. Sorafenib inhibited the EMT of HCC cells after insufficient 
RFA, and may be used to prevent the progression of HCC after RFA.

\section{Additional files}

\section{Additional file 1: NC3Rs ARRIVE Guidelines Checklist. (DOCX $664 \mathrm{~kb}$ ) \\ Additional file 2: The supplementary data. (DOCX $4641 \mathrm{~kb}$ )}

\section{Abbreviations}

BCA: Bicinchoninic acid; DMEM: Dulbecco's modified Eagle medium; ELISA: Enzyme-linked immunosorbent assay; EMT: Epithelial-mesenchymal transition; FBS: fetal bovine serum; HCC: hepatocellular carcinoma; MTT: 3-(4, 5-dimethylthiazol-2-yl)-2, 5-diphenyltetrazolium bromide; RFA: insufficient radiofrequency ablation.

\section{Competing interests}

The authors declare no competing interests.

\section{Authors' contributions}

SYD, JK and FDK carried out the experiments and drafted the manuscript. JGK, LJ and BP participated in the sequence alignment. LMZ and WBS conceived the study and coordination and helped to draft the manuscript. JG and LC participated in the design of the study. All authors read and approved the final manuscript.

\section{Acknowledgments}

This work was supported by Grants 320675007131 and 32067501207 from the Dr. Jieping Wu Medical Foundation, and the Program for Medical Key Discipline of Shijingshan District, Beijing. This work was also supported by Grants 2011CB503900 from "973" National S\&T Major Project, Grants $81170101,81370235,81572957$ and 81502650 from the National Natural Science Foundation of China, and Grants 7122106 from the Natural Science Foundation of Beijing, China. The authors would also like to thank Bayer Pharmaceuticals for providing the reagent.

\section{Author details}

${ }^{1}$ Department of Hepatobiliary Surgery, Beijing Chao-yang Hospital, Capital Medical University, Beijing 100043, China. ${ }^{2}$ School of Basic Medical Sciences, and Key Laboratory of Molecular Cardiovascular Sciences of Ministry of Education, The Institute of Cardiovascular Sciences and Institute of Systems Biomedicine, Peking University Health Science Center, Beijing 100191, China. ${ }^{3}$ Department of Neurobiology, School of Basic Medical Sciences, The Neuroscience Research Institute, Peking University, Beijing 100191, China. ${ }^{4}$ Key Laboratory for Neuroscience, Ministry of Education/National Health and Family Planning Commission, Peking University, Beijing 100191, China. ${ }^{5}$ The 8th Department of Orthopaedics, Affiliated Mindong Hospital of Fujian Medical University, Fujian 355000, China.

Received: 8 August 2014 Accepted: 20 November 2015 Published online: 30 November 2015

\section{References}

1. Forner A, Llovet JM, Bruix J. Hepatocellular carcinoma. Lancet. 2012; 379(9822):1245-55.

2. Lee DH, Lee JM, Lee JY, Kim SH, Yoon JH, Kim YJ, et al. Radiofrequency ablation of hepatocellular carcinoma as first-line treatment: long-term results and prognostic factors in 162 patients with cirrhosis. Radiology. 2014;270(3):900-9.

3. Nijkamp MW, van der Bilt JD, de Bruijn MT, Molenaar IQ, Voest EE, van Diest PJ, et al. Accelerated perinecrotic outgrowth of colorectal liver metastases following radiofrequency ablation is a hypoxia-driven phenomenon. Ann Surg. 2009:249(5):814-23.

4. Seki T, Tamai T, Ikeda K, Imamura M, Nishimura A, Yamashiki N, et al. Rapid progression of hepatocellular carcinoma after transcatheter arterial chemoembolization and percutaneous radiofrequency ablation in the primary tumour region. Eur J Gastroenterol Hepatol. 2001;13(3):291-4.

5. Ruzzenente A, Manzoni GD, Molfetta M, Pachera S, Genco B, Donataccio M, et al. Rapid progression of hepatocellular carcinoma after Radiofrequency Ablation. World journal of gastroenterology : WJG. 2004;10(8):1137-40.
6. Shiozawa K, Watanabe M, Takahashi M, Wakui N, lida K, Sumino Y. Analysis of patients with rapid aggressive tumor progression of hepatocellular carcinoma after percutaneous radiofrequency ablation. Hepatogastroenterology. 2009;56(96):1689-95.

7. Obara K, Matsumoto N, Okamoto M, Kobayashi M, Ikeda H, Takahashi H, et al. Insufficient radiofrequency ablation therapy may induce further malignant transformation of hepatocellular carcinoma. Hepatol Int. 2008;2(1):116-23.

8. Ke S, Ding XM, Kong J, Gao J, Wang SH, Cheng Y, et al. Low temperature of radiofrequency ablation at the target sites can facilitate rapid progression of residual hepatic VX2 carcinoma. J Transl Med. 2010;8:73.

9. Kong J, Kong J, Pan B, Ke S, Dong S, Li X, et al. Insufficient radiofrequency ablation promotes angiogenesis of residual hepatocellular carcinoma via HIF-1alpha/NEGFA. PLoS One. 2012;7(5), e37266.

10. Kong J, Kong L, Kong J, Ke S, Gao J, Ding X, et al. After insufficient radiofrequency ablation, tumor-associated endothelial cells exhibit enhanced angiogenesis and promote invasiveness of residual hepatocellular carcinoma. J Transl Med. 2012;10:230.

11. Dong S, Kong J, Kong F, Kong J, Gao J, Ke S, et al. Insufficient radiofrequency ablation promotes epithelial-mesenchymal transition of hepatocellular carcinoma cells through Akt and ERK signaling pathways. J Transl Med. 2013;11:273.

12. Yoshida S, Kornek M, Ikenaga N, Schmelzle M, Masuzaki R, Csizmadia E, et al. Sublethal heat treatment promotes epithelial-mesenchymal transition and enhances the malignant potential of hepatocellular carcinoma. Hepatology. 2013;58(5):1667-80.

13. De Craene B, Berx G. Regulatory networks defining EMT during cancer initiation and progression. Nat Rev Cancer. 2013;13(2):97-110.

14. Chen YL, Lv J, Ye XL, Sun MY, Xu Q, Liu CH, et al. Sorafenib inhibits transforming growth factor beta1-mediated epithelial-mesenchymal transition and apoptosis in mouse hepatocytes. Hepatology. 2011;53(5):1708-18.

15. Nagai T, Arao T, Furuta K, Sakai K, Kudo K, Kaneda H, et al. Sorafenib inhibits the hepatocyte growth factor-mediated epithelial mesenchymal transition in hepatocellular carcinoma. Mol Cancer Ther. 2011;10(1):169-77.

16. Chen YL, Zhang X, Bai J, Gai L, Ye XL, Zhang L, et al. Sorafenib ameliorates bleomycin-induced pulmonary fibrosis: potential roles in the inhibition of epithelial-mesenchymal transition and fibroblast activation. Cell death \& disease. 2013;4, e665

17. Zhang J, Chen YL, Ji G, Fang W, Gao Z, Liu Y, et al. Sorafenib inhibits epithelial-mesenchymal transition through an epigenetic-based mechanism in human lung epithelial cells. PLoS One. 2013;8(5), e64954.

18. Feng YX, Wang T, Deng YZ, Yang P, Li JJ, Guan DX, et al. Sorafenib suppresses postsurgical recurrence and metastasis of hepatocellular carcinoma in an orthotopic mouse model. Hepatology. 2011;53(2):483-92.

19. Kilkenny C, Browne WJ, Cuthill IC, Emerson M, Altman DG. Improving bioscience research reporting: the ARRIVE guidelines for reporting animal research. PLoS Biol. 2010;8(6), e1000412.

20. Chang RM, Yang H, Fang F, Xu JF, Yang LY. miR-331-3p promotes proliferation and metastasis of hepatocellular carcinoma by targeting PHLPP. Hepatology. 2014

21. Liu L, Dai Y, Chen J, Zeng T, Li Y, Chen L, et al. Maelstrom promotes hepatocellular carcinoma metastasis by inducing epithelial-mesenchymal transition by way of Akt/GSK-3beta/Snail signaling. Hepatology. 2014;59(2): 531-43.

22. Bao L, Yan Y, Xu C, Ji W, Shen S, Xu G, et al. MicroRNA-21 suppresses PTEN and hSulf-1 expression and promotes hepatocellular carcinoma progression through AKT/ERK pathways. Cancer Lett. 2013;337(2):226-36.

23. Rodon J, Dienstmann R, Serra V, Tabernero J. Development of PI3K inhibitors: lessons learned from early clinical trials. Nat Rev Clin Oncol. 2013;10(3):143-53.

24. Porta C, Paglino C, Mosca A. Targeting PI3K/Akt/mTOR Signaling in Cancer. Frontiers in oncology. 2014;4:64.

25. Wilhelm S, Carter C, Lynch M, Lowinger T, Dumas J, Smith RA, et al. Discovery and development of sorafenib: a multikinase inhibitor for treating cancer. Nat Rev Drug Discov. 2006;5(10):835-44.

26. Chen KF, Yu HC, Liu TH, Lee SS, Chen PJ, Cheng AL. Synergistic interactions between sorafenib and bortezomib in hepatocellular carcinoma involve PP2A-dependent Akt inactivation. J Hepatol. 2010:52(1):88-95.

27. Gedaly R, Angulo P, Hundley J, Daily MF, Chen C, Koch A, et al. Pl-103 and sorafenib inhibit hepatocellular carcinoma cell proliferation by blocking Ras/ Raf/MAPK and PI3KAKT/mTOR pathways. Anticancer Res. 2010;30(12):4951-8.

28. Shimizu S, Takehara T, Hikita H, Kodama T, Tsunematsu H, Miyagi T, et al. Inhibition of autophagy potentiates the antitumor effect of the multikinase 
inhibitor sorafenib in hepatocellular carcinoma. International journal of cancer Journal international du cancer. 2012;131(3):548-57.

29. Fukuda H, Numata K, Moriya S, Shimoyama Y, Ishii T, Nozaki A, et al. Hepatocellular Carcinoma: Concomitant Sorafenib Promotes Necrosis after Radiofrequency Ablation-Propensity Score Matching Analysis. Radiology. 2014;131640.

30. Li Y, Zheng YB, Zhao W, Liu B, Hu BS, He X, et al. Sorafenib in combination with transarterial chemoembolization and radiofrequency ablation in the treatment for unresectable hepatocellular carcinoma. Med Oncol. 2013;30(4):730.

31. Xu M, Xie XH, Xie XY, Xu ZF, Liu GJ, Zheng YL, et al. Sorafenib suppresses the rapid progress of hepatocellular carcinoma after insufficient radiofrequency ablation therapy: an experiment in vivo. Acta Radiol. 2013; 54(2):199-204

32. Boudou-Rouquette P, Narjoz C, Golmard JL, Thomas-Schoemann A, Mir O, Taieb F, et al. Early sorafenib-induced toxicity is associated with drug exposure and UGTIA9 genetic polymorphism in patients with solid tumors: a preliminary study. PLoS One. 2012;7(8), e42875.

33. Kong J, Kong F, Gao J, Zhang Q, Dong S, Gu F, et al. YC-1 enhances the anti-tumor activity of sorafenib through inhibition of signal transducer and activator of transcription 3 (STAT3) in hepatocellular carcinoma. Mol Cancer. 2014;13:7.

\section{Submit your next manuscript to BioMed Central and we will help you at every step:}

- We accept pre-submission inquiries

- Our selector tool helps you to find the most relevant journal

- We provide round the clock customer support

- Convenient online submission

- Thorough peer review

- Inclusion in PubMed and all major indexing services

- Maximum visibility for your research 